\title{
Priorités de la recherche forestière au Canada en 1990
}

\author{
Survol préparé pour le Conseil canadien des ministres des Forêts par le Conseil consul- \\ tatif de la recherche forestière du Canada en collaboration avec les comités consultatifs \\ de la recherche forestière des provinces et des territoires \\ Ottawa, Juillet 1990
}

\section{Résumé}

En 1987, le Conseil consultatif de la recherche forestière du Canada (CCRFC) a proposé une enquête annuelle, à la grandeur du Canada, sur les priorités de la recherche et sur les questions en devenir dont les résultats seraient présentés au Conseil canadien des ministres des Forêts (CCMF). La proposition a été acceptée comme une façon d'améliorer le dialogue sur la recherche, de même que la coordination et l'application de cette dernière. Les comités consultatifs de la recherche forestière des provinces et des territoires ont contribué au premier survol présenté au CCMF en octobre 1989 et publié dans le Forestry Chronicle de décembre 1989. Le présent rapport, le deuxième, se fonde sur la contribution des groupes consultatifs des provinces et des territoires fournie au début de 1990 .

La recherche forestière vise souvent le long terme, mais ses priorités évoluent. Le rapport énumère 23 nouveaux sujets de recherche. Plusieurs ont été cernés comme questions en devenir l'année dernière. Beaucoup des nouveaux sujets sont groupés sous une nouvelle rubrique intitulée POLITIQUE ET ÉCONOMIE FORESTIERES ou sous la rubrique ÉCOLOGIE et ils soulignent la nécessité d'études socio-économiques, de modèles de prédiction, de méthodes d'aide à la décision en ce qui concerne l'utilisation des sols et des ressources. Ils soulignent également les inquiétudes face aux modifications climatiques. Cette insistance sur les nouvelles questions traduit le fait que les aménagistes de l'industrie et des administrations publiques sont de plus en plus à l'écoute des attitudes du public pour ce qui est des questions de ressources et d'environnement. Ces attitudes sont de plus en plus affirmées avec la vogue montante de la notion de développement durable. En même temps, les préoccupations plus traditionnelles de la protection, de la récolte et de la régénération de la forêt conservent leur position prédominante dans les priorités de la recherche.

Les questions de politique et d'économie qui ont besoin de l'attention des chercheurs sont:

- les modèles de prédiction de l'offre et de la demande appliqués aux bois et à d'autres valeurs;

- des études socio-économiques élargies sur les conséquences des nouvelles politiques;

- les facteurs économiques des boisés privés;

- les méthodes de prise de décision sur l'utilisation des terres;

- les nouveaux régimes de propriété et les nouvelles modalités de vente du bois.

L'aménagement intégré des ressources forestières a besoin que l'on effectue de la recherche sur:

- l'aménagement de toutes les ressources: faune, loisir, bois, etc.;

- le fonctionnement des écosystèmes;
- les critères de décision et les systèmes d'information.

Les priorités de la recherche sur l'environnement comprennent:

- les effets de l'aménagement forestier et de la récolte sur l'écosystème forestier;

- les effets, sur la forêt, de la pollution atmosphérique et des modifications climatiques;

- le devenir des pesticides appliqués sur la forêt;

- le reboisement des stations contaminées;

- les effets des résidus de pesticide sur le matériel de plantation.

Les priorités de la recherche sur les ravageurs forestiers vont:

- aux solutions de rechange aux méthodes chimiques de lutte;

- aux méthodes améliorées d'évaluation du risque et de lutte;

- aux travaux sur des ravageurs précis tels que la tordeuse des bourgeons de l'épinette;

- aux ravageurs des pépinières et des jeunes peuplements;

- aux méthodes d'évaluation des dégâts;

- aux techniques de pulvérisation et à la prévention de la dérive des pesticides.

En raison des nombreux incendies de forêt, en 1989, la priorité a été accordée à la recherche sur:

- la prédiction des feux de friches, leur détection et leur extinction;

- l'amélioration et l'application des systèmes intégrés de lutte contre les incendies;

- l'écologie du feu.

En sylviculture, la régénération forestière et les soins culturaux bénéficient d'une priorité élevée pour la recherche, laquelle est particulièrement nécessaire sur:

- l'amélioration de la qualité des matériels de plantation;

- la lutte contre la végétation adventice;

- l'amélioration des arbres;

- la gestion des semences et des vergers à graines;

- la régénération des peupliers et des trembles hybrides;

- la réduction des coûts et l'accroissement de l'efficacité;

- l'amélioration des données sur l'accroissement, le rendement et les stations;

- la culture de feuillus de très bonne qualité dans l'est du Canada.

Les besoins de la recherche sur les feuillus forestiers concernant:

- les feuillus sous-utilisés;

- la qualité du bois récolté dans les forêts de seconde venue;

- l'amélioration de la fabrication des pâtes, des papiers et des produits en bois massif;

- l'invention de produits de valeur supérieure. 
Les grandes questions en puissance susceptibles de modifier les priorités, dans l'avenir, s'articulent autour:

- de la participation croissante du public dans l'aménagement des ressources;

- de la vogue croissante de la notion de développement durable;

- de l'engagement insuffisant du Canada dans la recherche-développement;

- du recyclage et de ses répercussions, tant sur l'aménagement forestier que sur les produits que l'on en tire;

- l'engagement croissant dans l'aménagement intégré des ressources forestières.

On s'inquiète également du fait que la vogue de l'écologie puisse détourner les priorités de la recherche de sujets moins brillants, mais néanmoins importants.

L'importance des Ententes sur la mise en valeur des ressources forestières pour accroître la recherche sur la forêt est universellement reconnue, de même que l'importance de leur reconduction.

Dans la plupart des provinces, il existe des organismes consultatifs sur la recherche, même si dans une ou deux des provinces, les progrès n'ont pas été rapides. Les programmes actuels de recherche sont considérés, dans la plupart des cas, comme scientifiquement et techniquement solides. L'insuffisance du financement continue d'inquiéter.

\section{Introduction}

En 1987, le Conseil consultatif de la recherche forestière du Canada (CCRFC) a proposé au Conseil canadien des ministres des Forêts (CCMF) que chaque province et territoire établisse un organisme consultatif unique sur la recherche forestière et que le CCRFC prépare l'examen annuel des priorités et des préoccupations cernées par ces groupes consultatifs.

La proposition a été acceptée. Les ministres étaient d'avis que le survol des préoccupations régionales et des priorités de la recherche en matière de foresterie serait à l'avantage du dialogue, qu'il améliorerait la coordination de la recherche et qu'il aiderait à l'application des résultats. Le premier rapport a été présenté au CCMF en octobre 1989 et publié dans le Forestry Chronicle de décembre 1989. C'était la première fois que l'on tentait de recenser et de synthétiser les priorités de la recherche en foresterie ainsi que les préoccupations dans ce domaine partout au pays; les renseignements avaient été réunis à la fin de 1988 et au début de 1989 par les groupes consultatifs des provinces et des territoires. Le présent rapport, le deuxième, se fonde sur des renseignements fournis au CCRFC à la fin de 1989 et au début de 1990.

La marche suivie dans la préparation du premier rapport a également servie pour le deuxième. Chaque groupe consultatif a préparé un bref compte rendu ordonné selon les rubriques suivantes:

- les priorités de la recherche forestière;

- les préoccupations naissantes en matière de foresterie et dans des domaines connexes susceptibles d'affecter les priorités de la recherche;

- les occasions nouvelles susceptibles d'influer sur les priorités de la recherche;

- Ia situation du processus de consultation en matière de recherche forestière;

- l'à-propos des programmes actuels de recherche.
Cette documentation a été envoyée au secrétaire exécutif du CCRFC qui en a prépare une ébauche pour examen à la réunion mixte du CCRFC et des représentants des provinces et des territoires. Elle a alors été revisée pour être soumise au Conseil des ministres des Forêts.

Les renseignements sur les priorités de la recherche ont été ordonnés en tableaux, selon la provenance et le sujet, puis groupés sous sept grands titres: politique et économie forestières (nouveau titre en 1990); aménagement intégré des ressources forestières; écologie; protection contre les ravageurs; protection contre les incendies; sylviculture et aménagement; produits forestiers (tableau 1).

La plupart des sondés ont choisi de présenter leurs renseignements sous forme de priorités générales de la recherche, sans commenter sur la nécessité d'efforts supplémentaires. D'autres ont toutefois cerné de façon précise les domaines où des travaux supplémentaires étaient considérés comme nécessaires. Ces sujets ont été identifiés au tableau 1 par l'adjonction de l'abréviation «t - (pour travaux supplémentaires) devant la rubrique pertinente.

Les renseignements sur les motifs nouveaux de préoccupation ainsi que sur les occasions nouvelles ont été soumis à la même analyse, mais les renseignements ont été réunis dans une liste intitulée Questions en devenir (tableau 2). Si un sujet avait été mentionné comme priorité de la recherche par au moins un sondé, puis comme nouveau motif de préoccupation ou comme occasion nouvelle par d'autres, les renvois à cette question ont été réunis dans le tableau des priorités de la recherche (tableau 1).

\section{Évolution des priorités de recherche}

En tout, 23 sujets de recherche ont été identifiés pour la première fois dans le présent rapport. Un sondé a proposé l'adjonction d'un nouveau grand titre, POLITIQUE ET ÉCONOMIE FORESTIERES, et comme cinq des nouveaux sujets se plaçaient dans cette catégorie, nous avons acquiescé. Cinq des nouveaux sujets portaient sur l'écologie, trois sur la protection contre les ravageurs et trois encore sur les produits forestiers. Ainsi, quatre domaines généraux, la politique et l'économie, l'environnement, la protection contre les ravageurs et les produits forestiers attirent actuellement l'attention, probablement, du moins dans le cas des trois premiers, parce que ce sont des sujets souvent d'actualité, qui préoccupent beaucoup le public et l'industrie forestière. Il va sans dire, toutefois, que les autres sujets antérieurement identifiés, tels que l'aménagement intégré et polyvalent, l'amélioration des arbres et la régénération forestière, conservent leur priorité.

D'intérêt et d'importance immédiats sont les 10 sujets relevés pour la première fois cette année par au moins deux groupes consultatifs. Ces sujets sont énumérés plus loin, sous le grand titre auquel ils se rattachent, le groupe qui les a proposés étant identifié après chacun. Certains de ces sujets avaient été considérés l'an dernier comme motifs nouveaux de préoccupation ou comme occasions nouvelles.

\section{Politique et économie}

- Modèles de prévision de l'offre et de la demande pour le bois et les autres valeurs que le bois (N.-B., -P.E., Qc, Ont., Man., C.-B., T.N.-O.). (La question avait été perçue comme nouvelle pour ce qui concerne la demande d'autres utilisations de la forêt). 


$\begin{array}{cccc}\text { T.-N. } & \text { N.-E. } & \text { N.-B. } & \text { I.-P.-E. } \\ + & + & + & +\end{array}$

POLITIQUE ET ÉCONOMIE, GÉNÉRALITÉS (NOUV. RUBRIQUE) $\mathrm{N}$ - Modèles de prévision, offre et demande, bois et autres ressources $\mathrm{T}$ - Études socio-économiques

$\mathrm{N}-$ Facteurs économiques des boisés privés

$\mathrm{N}$ - Encouragement aux investissements privés en sylviculture

$\mathrm{N}$ - Méthode d'aide à la décision concernant l'occupation des sols

- Amélioration des transferts de technologie

- Etudes de marketing

$\mathrm{N}$ - Nouveaux régimes de propriété et nouvelles modalités de vente du bois

AMÉNAGEMENT INTÉGRÉ DES RESSOURCES FORESTIERES, GÉNÉRALITÉS

T - Aménagement intégré du bois et des autres ressources

$\mathrm{T}$ - Fonctionnement de l'écosystème

$\mathrm{T}-$ Critères de décision et systèmes d'information

$\mathrm{T}-$ Hydrologie et drainage

\section{ÉCOLOGIE, GÉNÉRALITÉS}

$\mathrm{T}$ - Effets de l'aménagement forestier et de la récolte

$\mathrm{N}$ - Effets des changements climatiques

- Polluants atmosphériques

$\mathrm{N}$ - Devenir des pesticides appliqués sur les forêts

$\mathrm{N}$ - Reboisement des stations contaminées

$\mathrm{N}$ - Effets des procédés de fabrication

$\mathrm{T}$ - Résidus dans les matériels de pépinières

$\mathrm{N}$ - Effets de la monoculture

PROTECTION CONTRE LES RAVAGEURS, GÉNÉRALITÉS

$T$ - Méthodes de lutte de rechange

$\mathrm{T}$ - Evaluation et gestion du risque

- Études de ravageurs spécifiques

$\mathrm{T}$ - Maladies des pépinières et des jeunes peuplement

$\mathrm{T}$ - Évaluation des dommages

$\mathrm{T}$ - Homologation des pesticides dynamique

- Dynamique des populations de ravageurs

$\mathrm{T}$ - Techniques de pulvérisation et dérive, y compris du B.t.

$\mathrm{N}$ - Évaluation des applications de pesticides

$\mathrm{N}$ - Meilleures données sur la protection phytosanitaire

- Mécanismes de résistance aux maladies

$\mathrm{N}$ - Ravageurs des vergers à graines

PROTECTION CONTRE LES INCENDIES, GÉNÉRALITÉS

$\mathrm{T}$ - Prédiction et extinction des feux de friches

$\mathrm{T}$ - Systèmes intégrés de lutte contre les incendie

- Écologie du feu

- Évaluation et gestion du risque

- Effets socio-économiques

- Brûlage dirigé

$\mathrm{N}-$ Le feu et le pergélisol
$+$

$+$

$+$

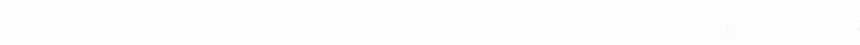

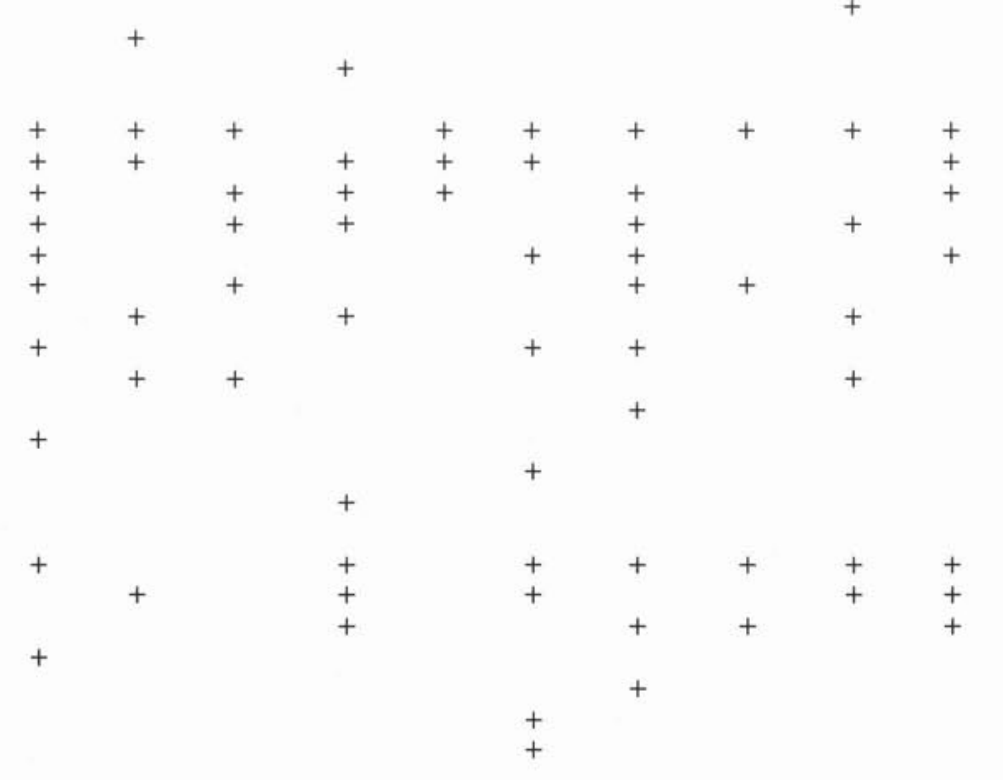


AMÉLIORATION DES ARBRES - GÉNÉRALITÉS

$\mathrm{T}$ - Amélioration des arbres

$\mathrm{T}$ - Biotechnologies

$\mathrm{T}$ - Gestion des semences

$\mathrm{T}$ - Aménagement des vergers à graines

$\mathrm{T}-$ Essais d'essences exotiques

SYSTÈMES DES RÉGÉNÉRATION, GÉNÉRALITÉS

$\mathrm{T}$ - Établissement de plantations

$\mathrm{T}$ - Qualité du matériel de plantation

$\mathrm{T}$ - Lutte contre la végétation adventice

$\mathrm{T}-$ Soins culturaux des nouvelles forets

- Méthodes naturelles de régénération

$\mathrm{N}$ - Régénération de peuplier faux-tremble et du peuplier hybride

- Ensemencement direct

$\mathrm{N}$ - Régénération du pin blanc

- Gestion des pépinières

DENDROMÉTRIE, GÉNÉRALITÉS

$\mathrm{T}$ - Accroissement, rendement, peuplements aménagés et peuplements naturels

$\mathrm{T}-$ Classement des stations

- Productivité des station

- Méthodes d'inventaire

$\mathrm{X}$ - Construction des courbes stationnelles

$\mathrm{X}$ - Inventaire forestier relativement aux produits finis

AMÉNAGEMENT FORESTIER, AUTRES QUESTIONS (GÉNÉRALITÉS)

$\mathrm{T}$ - Réduction des coûts de la sylviculture et mécanisation

$\mathrm{N}$ - Gestion des feuillus de qualité

$\mathrm{T}$ - Récolte, transport et solutions de rechange à la coupe à blanc

$\mathrm{N}$ - Mortalité des vielles épinettes croissant en situation exposée

$X$ - Aménagement des petits boisés

$\mathrm{X}$ - Aménagement pastoral

PRODUITS, GÉNÉRALITÉS

- Feuillus sous-utilisés

T - Propriétés du bois tiré des forets nouvelles

$\mathrm{N}$ - Produits à valeur ajoutée plus grande

- Carie et bois de rebut chez les feuillus

$\mathrm{N}$ - Transformation en produits massifs du bois

$\mathrm{N}$ - Nouveaux produits du génie du bois

$\mathrm{T}$ - Transformation en pâtes et papiers

$\mathrm{X}$ - Produits composites

$\mathrm{X}$ - Caractérisation des résidus

$\mathrm{X}-$ Produits chimiques

Nota bene: $\mathrm{N}$ - Signifie une nouvelle rubrique en 1990; T - que les travaux supplémentaires son exigés dans ce domaine: X - que la rubrique sera supprimée des rapports à venir. à moins d'un renouvellement de l'intérêt. 
Tableau 2. Questions en devenir (1990).

\begin{tabular}{|c|c|c|c|c|c|c|c|c|c|c|c|c|c|c|}
\hline & T.-N. & N.-E. & N.-B. & I.-P.-E. & QC & ONT. & MAN. & SASK. & ALB. & C.-B. & YUK. & T.N.-O. & $\begin{array}{c}\text { TOTAL } \\
1990\end{array}$ & $\begin{array}{c}\text { TOTAL } \\
1989 \\
\end{array}$ \\
\hline POLITIQUES ET ECONOMIE, GÉNÉRALITÉS & + & + & + & + & + & + & + & + & + & + & & + & 11 & 0 \\
\hline - Ententes de mise en valeur des ressources forestières et recherche & + & + & & + & + & & + & + & + & + & & + & 9 & 3 \\
\hline $\mathrm{N}$ - Émergence de la doctrine du développement durable & + & & & + & + & & + & & + & + & & & 6 & 0 \\
\hline $\mathrm{N}$ - Participation du public aux évaluations environnementales & + & & & + & & + & + & & + & + & & & 6 & 0 \\
\hline $\mathrm{N}$ - Engagement croissant envers l'aménagement intégré & + & + & & + & + & & + & & & + & & & 5 & 0 \\
\hline $\mathrm{N}$ - Mutation des valeurs sociales et conséquences pour la foresterie & & & & & & + & + & & & + & & & 3 & 0 \\
\hline - Faible engagement fédéral à l'égard de la foret & & & + & & & & & + & + & & & & 3 & 4 \\
\hline $\mathrm{N}$ - Amélioration des connaissances du public concernant la recherche & & & & & & & & & & & & & & \\
\hline forestière & + & & & & & + & & & & + & & & 3 & 0 \\
\hline $\mathrm{N}$ - Besoin de prédire les changements sociaux & + & + & & & & & & & & + & & & 3 & 0 \\
\hline $\mathrm{N}$ - Faible engagement envers la R-D au Canada & & + & & & + & & & & & + & & & 3 & 0 \\
\hline $\mathrm{N}$ - Recyclage & & + & & + & & + & & & & & & & 3 & 0 \\
\hline - Retard du système d'éducation & & & & + & & & & & & & & + & 2 & 2 \\
\hline - Plus de collaboration sur le plan de la recherche & + & & & & & & & & & & & + & 2 & 1 \\
\hline - Forets communautaires & & & & & & & & & & & & + & 1 & 1 \\
\hline - Demande accrue de bois & & & & & & & & & + & & & & 1 & 1 \\
\hline - Exigences a l'égard d'autre utilisations de la foret & & & & + & & & & & & & & & 1 & 1 \\
\hline - Création de Forets Canada & & & & & & & & & & & & + & 1 & 1 \\
\hline $\mathrm{N}$ - Politique concernant la production et l'offre en matière de forets & & & & & & & & & & & & & & \\
\hline et de bois & & & & & & + & & & & & & & 1 & 0 \\
\hline - Personnel mieux formé & + & & & & & & & & & & & & 1 & 1 \\
\hline - Sylviculture et régénération par l'industrie & + & & & & & & & & & & & & 1 & 2 \\
\hline - Priorités modifiées par les préoccupations écologiques & & & & & & + & & & & & & & 1 & 2 \\
\hline ENVIRONNEMENT, GÉNÉRALITÉS & + & + & & & & & & & & + & & & 3 & 2 \\
\hline $\mathrm{N}$ - Contamination des eaux souterraines par les pesticides & & + & & & & & & & & & & & 1 & 0 \\
\hline PROTECTION CONTRE LES RAVAGEURS, GÉNÉRALITÉS & + & & & & & & + & & & + & & & 3 & 1 \\
\hline $\mathrm{N}$ - Examen de processus fédéral d'homologation des pesticides & & & & & & & + & & & + & & & 2 & 0 \\
\hline SILVICULTURE ET AMÉMAGEMENT, GÉNÉRALITÉS & + & + & + & + & + & + & + & & + & + & & + & 10 & 7 \\
\hline $\begin{array}{l}\mathrm{N} \text { - Aménagement des forets sous-utilisées de feuillus et de peuple- } \\
\text { ments mélangés }\end{array}$ & + & & + & & & & + & & + & + & & & 5 & 0 \\
\hline - Utilisation des pesticides et des herbicides & + & + & & & & + & & & & + & & & 4 & 4 \\
\hline - Informatisation et systèmes experts & + & & & & + & + & & & & & & + & 4 & 1 \\
\hline - Sylviculture en vue de l'accroissement du rendement & & + & & & & & & & + & + & & & 3 & 3 \\
\hline $\mathrm{N}$ - Effets de la lutte contre la végétation adventice sur les arbres & & & & & & & & & & & & & & \\
\hline cultivés & & + & & + & & & & & & & & & 2 & 0 \\
\hline - Besoin d'une croissance plus rapide et sans entrave & + & & & & & & & & + & & & & 2 & 3 \\
\hline - Régénération insuffisant, à long terme & & & & & & & & & + & & & & 1 & 3 \\
\hline - Silviculture dans les stations de choix & & & & & & + & & & & & & & 1 & 2 \\
\hline PRODUITS, GÉNÉRALITÉS & & & & + & & + & & & + & & & & 2 & 1 \\
\hline $\mathrm{N}$ - Combustibles tirés de la biomasse ligneuse & & & & + & & & & & & & & & 1 & 0 \\
\hline
\end{tabular}

Nota bene: $\mathrm{N}$ - Signifie nouvelle rubrique en 1990. 
- Facteurs économiques des boisés privés (.-P.-É., Qc, Man.).

- Encouragements à l'investissement privé en sylviculture (.-P.-É., C.-B.).

- Méthodes pour aider aux décisions concernant l'occupation des sols (T.-N., C.-B.).

\section{Environnement}

- Effets des changements climatiques (Ont., Man., C.-B.). (question perçue comme nouvelle, l'année auparavant).

- Le devenir des pesticides appliqués sur les forêts (.-P.-É., Man.).

\section{Sylviculture et aménagement forestier}

- Régénération du tremble et du peuplier hybride (Man., Sask., C.-B.).

- Aménagement des feuillus de qualité (.-P.-É., Qc, Ont.). (Question antérieurement perçue comme nouvelle).

\section{Produits}

- Transformation en produits du bois massif (Qc, Alb.). (Question antérieurement perçue comme nouvelle pour ce qui concerne les petites billes).

- Produits à plus grande valeur ajoutée (Ont., Alb.). (Question antérieurement perçue comme nouvelle).

Beaucoup des nouveaux sujets de cette année ont été mentionnés par un seul sondé. N'en soyons pas surpris, les idées et les préoccupations nouvelles se manifestant souvent comme la conséquence d'un problème spécifique, localisé. Il est probable qu'elles gagneront du terrain. Leur importance ne saurait être niée, même si elles sont d'abord été perçues par un seul groupe. Ces sujets ont été ordonnés comme suit sous leur rubrique principale.

\section{Politique et économie}

- Nouvelles formes de régime foncier et nouvelles modalités de vente du bois (C.-B.).

\section{Environnement}

- Reboisement des stations contaminées (Man.).

- Effets des procédés de fabrication sur l'environnement (Alb.).

- Effets de la monoculture (.-P.-É.).

\section{Protection contre les ravageurs}

- Amélioration des renseignements sur la protection des plantes contre les ravageurs (T.-N.).

- Évaluation des applications de pesticides (Man.).

- Ravageurs des vergers à graines (.-P.-É.).

Protection contre les incendies

- Les incidents et le pergélisol (T.N.-O.).

\section{Sylviculture et aménagement}

- Régénération du pin blanc (.-P.É.).

- Mortalité des vieilles épinettes croissant en situation dégagée (.-P.-É.).

\section{Produits}

- Nouveaux produits du génie du bois (Alb.). (Sujet nouveau de préoccupation, l'an dernier, sous la rubrique Le bois dans la construction).

Onze sujets de recherche identifiés l'an dernier n'ont pas été mentionnés cette année et six d'entre eux ont été marqués comme à radier de la liste des priorités en raison du peu d'intérêt suscité. Les autres ont été ordonnés sous d'autres rubriques.

\section{Discussion générale sur les priorités de la recherche}

Un résultat très intéressant et significatif du sondage de cette année est l'intérêt marqué à l'égard de la recherche sur la politique et l'économie forestières. Le phénomène, du moins en partie, répond aux préoccupations écologiques, mais il montre également l'attention que consacrent de plus en plus les aménagistes de l'industrie et des administrations publiques aux attitudes du public sur toutes les questions touchant les ressources. La vogue de plus en plus grande de la notion de développement durable a amené le public à exprimer plus catégoriquement son opinion sur l'environnement, l'utilisation des ressources et la conservation. Les aménagistes réclament plus de travaux de recherche pour construire des modèles de la prédiction de l'offre et de la demande de bois ainsi qui des autres valeurs que le bois; de meilleurs moyens pour prendre des décisions sur les formes d'occupation du sol et davantage d'études socio-économiques, y compris celles qui visent à évaluer les valeurs sociales en mutation ainsi que leurs répercussions sur l'aménagement des ressources forestières.

L'environnement continue de conserver la priorité de la recherche dans la plupart des provinces. Les craintes au sujet de l'environnement et du développement durable sont au centre des préoccupations du public dans les questions touchant les ressources naturelles. En aménagement forestier, les sujets d'inquiétude concernant l'habitat de la faune, les valeurs naturelles, les forêts à maturité ou surâgés, la coupe à blanc et l'emploi de produits chimiques. Les aménagistes de l'industrie et des administrations publiques partagent ces inquiétudes, ce que traduisent les priorités cernées de la recherche. Par exemple, une forte priorité est accordée à la recherche visant à clarifier les effets des pratiques d'aménagement forestier et de récolte ainsi que de la pollution atmosphérique sur l'écosystème forestier. Dans la même veine, on réclame davantage de travaux sur le devenir des pesticides dans la forêt.

Dans la protection contre les ravageurs, qui, dans l'ensemble, reçoit une priorité élevée, on insiste beaucoup sur l'amélioration des méthodes d'évaluation du risque et des dommages ainsi que sur la recherche de solutions de rechange aux produits chimiques. Ces techniques améliorées sont considérées comme nécessaires pour parfaire les décisions prises dans des circonstances données contre les ravageurs et pour diffuser les méthodes biologiques efficaces à cette fin.

La forte priorité accordée à la recherche visant à améliorer l'aménagement intégré des ressources forestières traduit également le désir des forestiers de redorer, sur le plan écologique, le blason de l'aménagement, de façon à en retirer une large gamme d'avantages, tant pour le bois que pour les autres ressources.

Comme nous l'avons noté, la protection contre les ravageurs forestiers continue de conserver la priorité de la recherche, et, outre les sujets précités, on accorde de plus en plus d'importance à la recherche sur les ravageurs des pépinières et des jeunes peuplements, sur les techniques de traitement par pulvérisation et de prévention de la dérive ainsi qu'à l'homologation des nouveaux pesticides. Le besoin 
d'améliorer la qualité des renseignements disponibles pour aboutir à une protection phytosanitaire efficace des plantes a été identifié par un sondé. Certains ravageurs spécifiques ont été identifiés comme des sujets prioritaires, y compris, évidemment, la tordeuse des bourgeons de l'épinette. Cependant, plusieurs autres fléaux, d'importance plus locale, ont été mentionnés, par exemple la galle vésiculeuse de l'ouest, les scolytes de l'écorce et la carie des tiges.

En matière d'incendie, aucun nouveau sujet de recherche n'a été trouvé. En raison de la campagne difficile de 1989, où un nombre sans précédent d'incendies forestiers se sont déclarés au Canada, on réclame d'intensifier les efforts dans la prédiction et la détection des feux de friches ainsi que dans leur extinction et la mise au point et l'application de systèmes intégrés de riposte à l'incendie, sujets qui, évidemment, sont très étroitement apparentés.

Dans le domaine général de la sylviculture, la recherche visant l'amélioration de la régénération forestière et celle qui porte sur l'amélioration des arbres correspondent à la priorité la plus élevée. Le besoin d'améliorer la qualité du matériel de plantation et d'améliorer l'implantation, y compris la lutte contre la végétation adventice, a reçu une priorité très élevée. Comme nous l'avons fait remarquer auparavant, la recherche sur la gestion des pépinières a apparemment reçu une priorité plus faible cette année, mais le sujet semble avoir été repris dans la demande de plus de travaux de recherche pour améliorer la qualité du matériel de plantation. Les priorités de l'amélioration des arbres portent sur les biotechnologies, la gestion des semences et l'aménagement des vergers à graines ainsi que sur les essais d'essences exotiques.

Il existe un besoin de meilleurs renseignements sur les techniques de régénération du peupliers faux-trembles et des peupliers hybrides. La sylviculture de la forêt de seconde venue, dite également "nouvelle», a également été identifiée comme ayant besoin de travaux supplémentaires de recherche, tandis que la régénération naturelle a encore une fois été qualifiée d'importante par trois comités consultatifs. De même, trois provinces réclament plus de travaux sur la réduction des coûts et la mécanisation de la sylviculture de même que sur la récolte, le transport et les solutions de rechange à la coupe à blanc.

Plus de recherche est nécessaire pour améliorer les renseignements que l'on possède sur l'accroissement et le rendement, particulièrement, mais sans s'y limiter, en ce qui concerne les peuplements de seconde venue. Les problèmes connexes touchant la classification des stations possèdent également une priorité très haute dans beaucoup de régions. Enfin, pour ce qui est des priorités de la recherche sylvicole, la culture de feuillus de qualité a été identifiée comme une nouvelle priorité de la recherche cette année dans certaines parties de l'Est. Auparavant, elle avait été identifiée comme une question en devenir.

La recherche sur les produits forestiers conserve une priorité considérable, même si trois sujets relevés l'an dernier ne se retrouvent plus dans les réponses données cette année: les produits chimiques, les produits composites (considérer que les produits à valeur ajoutée englobent ces deux sujets) et la caractérisation des résidus. Suscitent cependant vraiment les inquiétudes les questions soulevées par les propriétés du bois croissant dans les forêts de seconde venue. Des travaux supplémentaires de recherche sont nécessaires pour évaluer ces propriétés et pour élaborer des procédés de transformation nouveaux et plus efficaces, tant pour les produits du bois massif que pour les pâtes et papier. Dans beaucoup de régions, le cubage considérable d'essences sous-utilisées de feuillus fait que la recherche d'emplois nouveaux, à forte valeur ajoutée, pour ces matériaux conserve la priorité.

Pour conclure sur les priorités, on fera remarquer que le transfert de technologies a été mentionné comme important dans une seule réponse cette année. Première vue, cela semble surprenant, à la lumière de la priorité élevée accordée à la question l'an dernier, mais celle-ci se fond de plus en plus dans le continuum recherche-développement-innovation. Il est vrai qui ce n'est pas à strictement parler une priorité de la recherche, mais plutôt un aspect important des applications de cette dernière.

\section{Préoccupations et occasions nouvelles qui peuvent influer sur les priorités}

En raison de la distinction entre les préoccupations et les occasions, qui n'est pas toujours claire en ce que les préoccupations ou les problèmes d'une personne peuvent constituer une occasion pour autrui, les renseignements sur les questions en devenir ont été groupés sous une seule rubrique cette année. Quatorze nouveaux sujets ont été cernés et neuf d'entre eux sont ordonnés sous le nouveau grand titre Politique et économie. Certains des nouveaux sujets n'ont été notés que par une ou deux sources, mais les quatre suivantes ont été mentionnées cinq ou six fois chacune:

- accroissement de la participation du public et évaluations environnementales (T.-N., -P.-É., Ont., Man., Alb., C.-B.);

- la nouvelle doctrine du développement durable (T.-N., -P.-É., Ont., Man., Alb., C.-B.);

- l'engagement croissant dans l'aménagement intégré des forêts (T.-N., N.-É., -P.-É., Man., C.-B.);

- l'aménagement des peuplements sous-utilisés de feuillus et des peuplements mélangés (T.-N., N.-B., Man, Alb., C.-B.). Les trois premières de ces nouvelles questions ont été mentionnées cinq fois au moins et elles sont étroitement reliées, ce qui traduit encore une fois le souci croissant dont font preuve les aménagistes pour l'intérêt public en général. Comme nous l'avons déjà relevé, cette tendance s'est également manifestée dans les réponses sur les priorités de la recherche. La désignation des essences sous-utilisées de feuillus à l'attention de la recherche, comme question en devenir, ne surprend pas. Il s'agit de l'une des dernières occassions d'expansion de l'économie fondée sur la forêt au Canada.

D'autre part, 13 sujets qui avaient été identifiés l'an dernier n'ont pas été mentionnés cette année comme questions en devenir, mais il faut y inclure huit sujets qui sont passés de la catégorie des questions en devenir à celles des priorités de la recherche, c'est-à-dire la gestion de la qualité des feuillus; les résidus de pesticides sur le matériel de pépinière; les changements climatiques; le bois dans la construction; la transformation des petites billes; la demande d'autres utilisations des forêts; les produits à valeur ajoutée; l'application des systèmes experts.

Les 10 autres nouveaux sujets sont énumérés ci-dessous sous leur grand titre respectif.

\section{Politique et économie}

- Les valeurs sociales en transformation et leurs effets sur la foresterie (Ont., Man., C.-B.). 
- Le faible engagement envers la recherche-développement au Canada où moins de $1 \%$ du PIB est consacré à la recherche (N.-É., Qc, C.-B.).

- L'importance d'essayer de prédire les changements sociaux plutôt que d'y réagir seulement (T.-N., N.-É., C.-B.).

- Les effets du recyclage sur la transformation des produits forestiers et sur l'aménagement de la ressource (N.-É., -P.-É., Ont.).

- L'importance d'améliorer les connaissances du public sur la recherche forestière et l'acquis de cette dernière (T.-N., Ont., C.-B.).

- Le besoin d'élaborer de nouvelles politiques concernant la production forestière et les réserves forestières (Ont.).

\section{Environnement}

- La contamination possible des eaux souterraines par les pesticides, de plus en préoccupante (N.-É.).

\section{Protection contre les ravageurs}

- L'examen du processus fédéral d'homologation des pesticides peut changer les usages ainsi que la disponibilité des pesticides (Man., C.-B.).

\section{Sylviculture et aménagement}

- Les effets du désherbage sur les arbres cultivés sont peu connus (N.-É., -P.-É.).

\section{Produits forestiers}

- Combustibles tirés de la biomasse ligneuse (.-P.-É.).

L'intérêt naturel à l'égard des questions que l'on vient de percevoir ne devrait pas faire perdre de vue un certain nombre de questions en devenir qui avaient déjà été perçues et qui sont confirmées par les réponses des sondés. Dans le domaine de la politique et de l'économie, on attache beaucoup d'importance aux Ententes sur la mise en valeur des ressources forestières, pour le financement de la recherche forestière. La lenteur de la reconduction des ententes est préoccupante pour ce qui touche l'engagement apparemment faible du gouvernement fédéral à l'égard des questions de la forêt en général. L'on craint encore que les préoccupations écologiques ne puissent dépouiller de leur priorité les questions importantes, mais moins populaires. Par exemple, l'intérêt actuel à l'égard des changements climatiques pourrait se faire au détriment de la recherche sur les soins culturaux des nouvelles plantations pour en tirer une productivité maximale, par exemple, ou de la recherche de moyens pour améliorer les pratiques sylvicoles dans les stations forestières de choix.

\section{Points sur le processus consultatif sur la recherche}

Les structures consultatives sont maintenant en place dans toutes les provinces et tous les territoires, même si elles peuvent varier dans leur caractère officiel et les secteurs qu'elles représentent. Il est clair qu'il existe une évolution vers les organes consultatifs sur la recherche unique, qui se reconnaissent entre eux et qui sont orientés vers les utilisateurs, dans chaque province et territoire, comme l'avait réclamé le Conseil des ministres des Forêts, en septembre 1987. Dans tous les cas, les objectifs sont de surveiller et de coordonner les programmes de recherche et d'orienter les besoins de cette dernière selon un ordre de priorité. Les détails, par province et territoire, sont donnés à l'annexe I.

\section{A-propos des programmes actuels de recherche}

En général, la recherche forestière au Canada est considérée comme scientifiquement et techniquement solide, mais dans certains cas elle semble mal coordonnée. Certains estiment que les chercheurs réagissent trop lentement aux demandes de changement dans l'orientation de leurs travaux ou de nouveaux travaux. Le financement fourni à la recherche est généralement considéré comme insuffisant ou cruellement parcimonieux, même si une réorientation et la coordination sont considérées comme presque aussi importantes que l'accroissement de financement. Même si le financement de la recherche en vertu des Ententes sur la mise en valeur des ressources forestières est trs̀ utile, on pense que le fait de trop compter sur cette source menace le financement de base qui est essentiel à la recherche à long terme dont a besoin la forêt.

\section{ANNEXE I}

\section{Structures consultatives dans les provinces et les territoires}

Terre-Neuve, un organisme consultatif qui existait depuis de nombreuses années, a été reconnu officiellement dans un protocole d'entente entre le Canada et la province en 1982. Le dernier protocole signé en 1988 prévoit l'apport officiel de l'industrie au Comité consultatif de la recherche forestière Canada - Terre-Neuve. Le comité est constitué de neuf membres, se réunit deux fois par année, et est coprésidé par des hauts fonctionnaires du Canada et de la province.

En Nouvelle-Écosse, on trouve deux comités. Un comité de travail, le comité consultatif de la recherche forestière en Nouvelle-Écosse, se réunit deux fois par année et possède des représentants des deux niveaux de gouvernement, de l'industrie, de l'université du Nouveau-Brunswick, de la Nova Scotia Christmas Tree Growers et des propriétaires de boisés. Outre son rôle consultatif à l'égard de la recherche, le comité établit les priorités et propositions de recherche destinées à être financées en vertu de l'Entente sur la mise en valeur des ressources forestières. Ses recommandations parviennent à un comité principal, le comité mixte Canada - Nouvelle-Écosse de la recherche forestière, constitué de huit membres recrutés egalement dans les deux administrations publiques. On envisage d'y admettre des représentants de l'industrie.

Au Nouveau-Brunswick, on compte également deux groupes. Le Comité consultatif de la recherche forestière du Nouveau-Brunswick est présidé par le sous-ministre des Ressources naturelles et de l'Énergie de la province. Constitué de 12 membres recrutés chez les cadres de l'industrie, des deux administrations publiques et des deux universités où se donnent des cours de foresterie dans la province, le comité se réunit de deux à quatre fois par année. Il fonctionne par le truchement d'une série de comités techniques qui orientent également la recherche financée en vertu de l'Entente sur la mise en valeur des ressources forestières. L'autre comité, le Comité directeur de la recherche forestière du Nouveau-Brunswick, est constitué en société et compte parmi ses membres le sous-ministre de la province, le directeur général régional de Forêts Canada ainsi que les directeurs 
généraux des entreprises de l'industrie forestière. Ce comité permet aux dirigeants du secteur forestier de participer à l'orientation de la recherche forestière dans la province.

Dans I'Ile-du-Prince-Édouard, le Comité consultatif de la recherche forestière est coprésidé par le directeur des opérations forestières du ministère provincial de l'Énergie et des Forêts ainsi que par le directeur de programme pour la Recherche dans la région des Maritimes de Forêts Canada. Il compte trois membres de la province et autant de Forêts Canada. Il se réunit deux fois l'an pour examiner les projets de recherche à financer en vertu de l'Entente sur la mise en valeur des ressources forestières et pour coordonner les autres travaux de recherche.

Le Conseil de la recherche forestière du Québec a été par décret en 1988 et est maintenant l'organisme officiel de consultation, de coordination et d'orientation des programmes de recherche-développement sur la forêt. Il est composé de groupes intéressés à la conduite et à l'application de la recherche forestière, chacun de ces groupes étant représenté par un ou deux membres auprès du Conseil, comme suit: le ministre de l'Énergie et des Ressources du Québec (1); Forêts Canada (1); l'Université Laval (1); l'Université du Québec (1); l'Association des industries forestières du Québec (2); l'Association des manufacturiers de bois de sciage du Québec (2); l'Association des propriétaires de boisés du Québec (2). Le Conseil est financé par les organismes participants, en relation directe avec le nombre de représentants qu'ils ont au conseil, disposition unique au Canada. Le Conseil définit les besoins de la recherche et ses priorités; il propose des organismes pour effectuer les travaux; il encourage la coordination de la recherche et du développement par voie expérimentale; il propose des moyens pour assurer la diffusion maximale des renseignements scientifiques et techniques; il encourage l'application et le transfert des innovations scientifiques et techniques dans la pratique. Des comités spéciaux ont été constitués comme suit: (1) comité des solutions de rechange des biotechnologies aux pesticides chimiques; (2) comité de la production de matériels de plantation; (3) comité de la R-D sur l'emploi des essences sous-utilisées.

Le Conseil de recherche forestière de l'Ontario, établi par décret en 1984, cerne les besoins de la recherchedéveloppement et recommande des priorités. Il est constitué d'un sous-ministre fédéral et provincial et d'un membre supplémentaire de chaque administration; de quatre cadres de l'industrie forestière et d'un président d'université. Son président est nommé par le ministre des Ressources naturelles. En 1987, le conseil a créé l'Ontario Forestry Research Committee (OFRC) et lui a confié le mandat de lui servir de comité technique. Ce comité est présidé par le viceprésident du Conseil et compte cinqu membres de l'industrie forestière, quatre du ministère des Ressources naturelles de la province et un membre de chacun des organismes suivants: région de l'Ontario de Forêts Canada, The Ontario Tree Improvement Council et les facultés de foresterie des Universités Lakehead et de Toronto. Les recommandations du Comités sur les priorités de la recherche et d'autres questions sont soumises au Conseil.

Au Manitoba, un protocole d'entente a présidé, en 1987, à la création du comité mixte de recherche constitué de deux représentants des deux gourvernements. Le comité examine les travaux en cours et détermine les nouveaux sujets qui exigent l'attention. Au cours des cinq dernières années, la recherche a été financée en vertu de l'Entente de mise en valeur des ressources forestières signée entre le Canada et le Manitoba. Un comité consultatif de la recherche forestière du Manitoba a été proposé, conformément à la décision du Conseil canadien des ministres des Forêts. Il se réunira au cours de l'été de 1990 et apportera une représentation élargie à l'examen des priorités de la recherche forestière dans la province.

Le Comité consultatif de la recherche forestière de la Saskatchewan est en gestation, en réponse à une recommandation du CCMF. Il comptera huit membres, comme suit: Saskatchewan (2); Canada (2); industrie forestière (2); faculté de foresterie de l'Université de l'Alberta (1); une autre université (1).

En réponse à une proposition du CCMF, l'Alberta a créé un conseil consultatif de la recherche forestière de l'Alberta en vertu de l'Alberta Forest Development Research Trust Fund Act. Le conseil donne son avis au ministre des Forêts, des Terres et de la Faune sur les priorités de la recherche, la coordination de cette dernière et les possibilités de financement. Il est présidé par le sous-ministre de la province et compte parmi ses membres le sous-ministre adjoint de la province et le sous-ministre ainsi que le directeur général régional de Forêts Canada. Le directeur exécutif-de l'Alberta Forest Products Association, le doyen de la faculté d'agriculture et de foresterie de l'Université de l'Alberta et le président du Conseil de recherche de l'Alberta en sont également membres de même que les directeurs généraux de neuf des entreprises qui fonctionnent en Alberta.

Le Comité consultatif de la recherche forestière de la Colombie-Britannique est le noyau consultatif qui donne avis sur la planification et les priorités de la recherche sur les ressources forestières. Il est constitué de représentants de Forêts Canada (1); du ministère des Forêts de la province (3), du ministère de l'Environnement de la province (1), des universités (1), du Conseil des sciences de la Colombie-Britannique (1), de l'Industrie forestière (4) et du public (1). Les comités consultatifs techniques et régionaux de la recherche informent le premier comité. Le rôle du comité consultatif de la recherche forestière de la Colombie-Britannique est:

- d'identifier et de classer les questions importantes exigeant l'attention des chercheurs, à la lumière de l'apport des comités techniques et des comités régionaux;

- d'examiner périodiquement le mandat des comités techniques pour faire en sorte que toutes les questions importantes possèdent leurs groupes d'examen et de planification technique;

- d'orienter les comités techniques (par le truchement de conseils ou de comités directeurs, s'il y a lieu) sur les besoins de la planification, des besoins pour les grandes questions ou les grandes volets de ces dernières, et sur la preparation des plans et les rapports d'étape;

- de communiquer les besoins et les priorités de la recherche ainsi que les plans endossés des comités techniques auprès des organismes de recherche pour étude et mise en oeuvre;

- de communiquer les besoins et les priorités de la recherche de la Colombie-Britannique au Conseil consultatif de la recherche forestière du Canada ainsi qu'au Comité consultatif des sciences et de la technolgie de Premier ministre. Le Comité consultatif de la recherche forestière du Yukon possède des représentants de Forêts Canada, du ministère des Ressources renouvelables du Yukon ainsi que de la 
Division des ressources forestières du ministre des Affaires indiennes et du Nord. Il se réunit deux fois l'an pour examiner les progrès de la recherche et les priorités des travaux à venir.

Les Territoires du Nord-Ouest ne possèdent pas d'organe consultatif officiel sur la recherche, mais ils participent au Comité consultatif régional principal de Forêts Canada qui fonctionne depuis quelques années afin de donner son avis général et son avis sur la recherche au directeur général de la région du Nord-Ouest de Forêts Canada. Les membres de ce comité sont recrutés dans la région et représentent les gouvernements des trois provinces des Prairies et des Territoires du Nord-Ouest, l'industrie forestière, les établissements d'éducation supérieure et les parcs nationaux.

\section{CIF/IFC Corporate Sustaining Members}

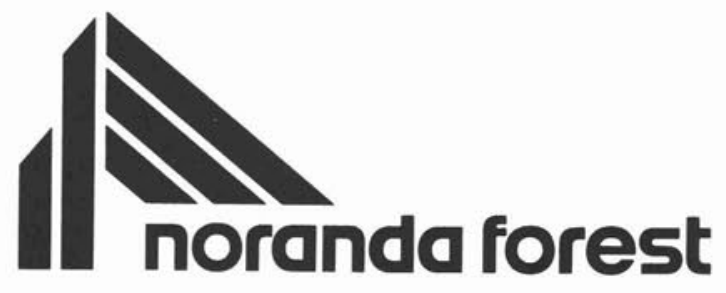

$\Lambda$

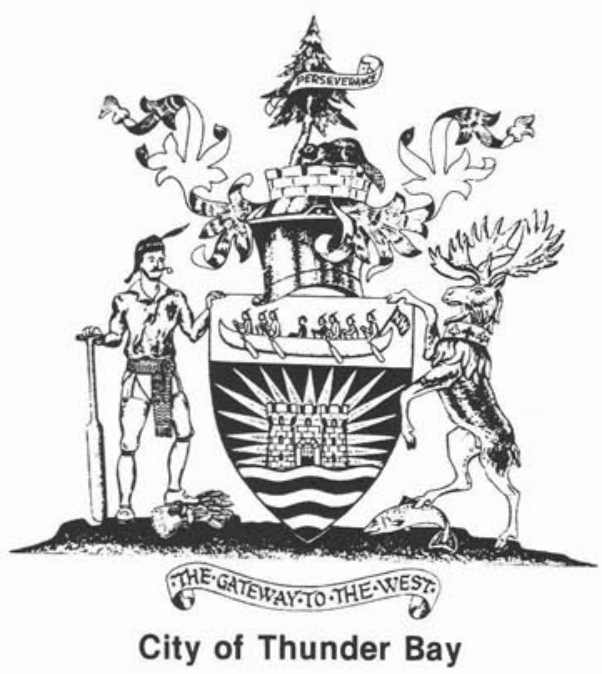

\section{STORA \\ Stora Forest Industries Limited}
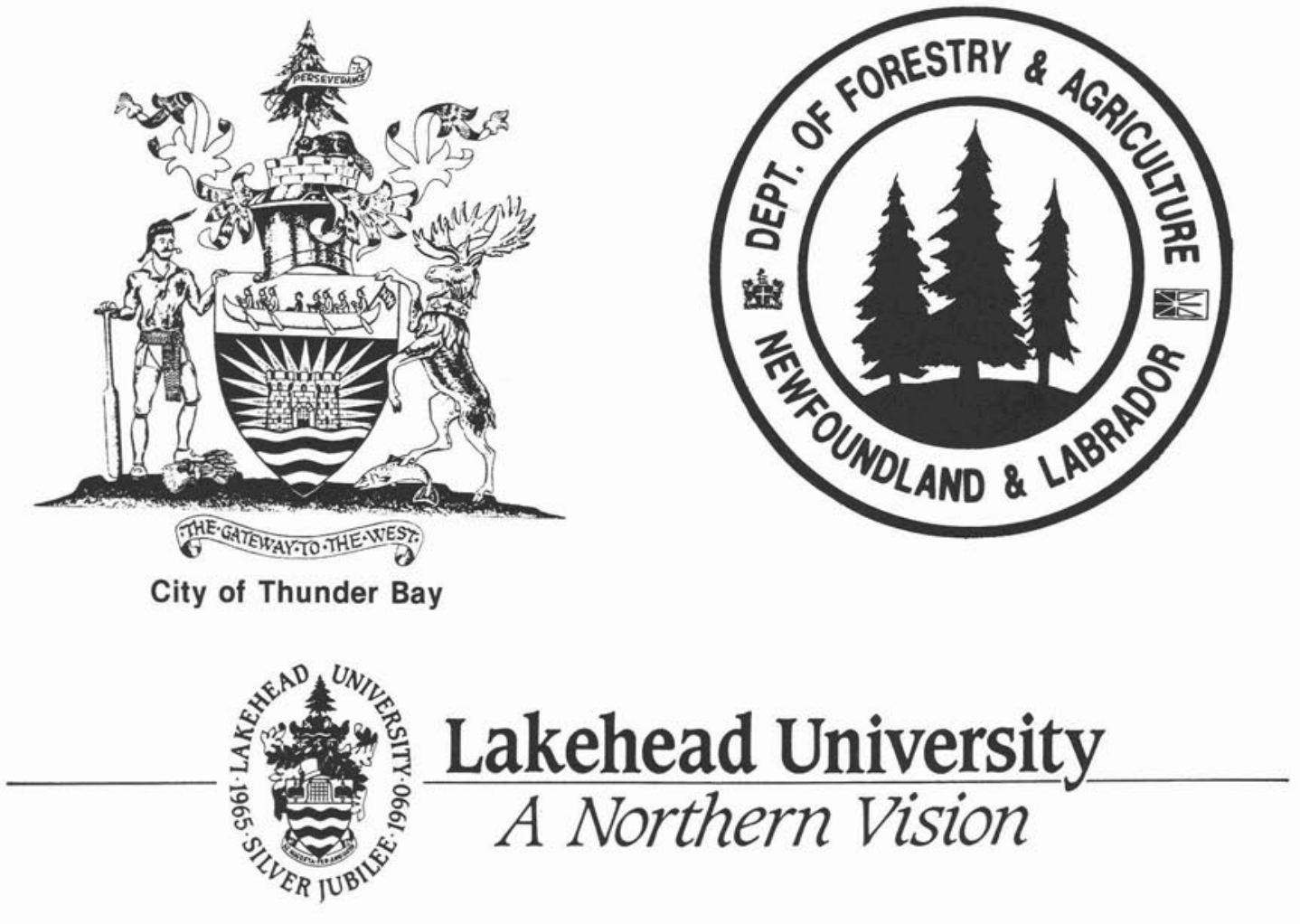